\title{
The Active Badge Location System
}

\author{
Roy Want ${ }^{1}$, Andy Hopper ${ }^{2}$, Veronica Falcão ${ }^{3}$ and Jonathan Gibbons ${ }^{4}$ \\ Olivetti Research Ltd. (ORL) \\ Cambridge, England
}

\begin{abstract}
A novel system for the location of people in an office environment is described. Members of staff wear badges that transmit signals providing information about their location to a centralized location service, through a network of sensors. The paper also examines alternative location techniques, system design issues and applications, particularly relating to telephone call routing. Location systems raise concerns about the privacy of an individual and these issues are also addressed.
\end{abstract}

\section{Introduction}

Efficient location and coordination of staff in any large organization is a difficult and recurring problem. Hospitals, for example, may require up-to-date information about the location of staff and patients, particularly when medical emergencies arise. In an office building, a receptionist is usually responsible for determining the location of staff members; in some organizations, publicaddress systems are provided to help a receptionist locate employees but, more frequently, a telephone is used to contact all the possible locations at which the required person might be found. These solutions can cause a great deal of irritation and disruption to other employees; a solution that provides direct location information is more desirable.

Location information for office staff that is available in a computer-readable format can also be used to improve the operation of the office telephone system. Integration of telephone systems with computer systems is also important in the development of the automated office. Much work has already been undertaken integrating digital voice and computer data into a single network [4], but there has been less commercial effort invested in improving the telephone interface. Although these interfaces are functionally sophisticated, they are cryptic and their operation is difficult to remember. The features most commonly used by PBX clients are 'call transfer' and 'call forward'. In most cases the execution of these features could be automated by the PBX if it had information about the current location of its clients.

\section{Existing Location Systems}

The conventional solution used for personnel location is the 'pager system'. In order to locate someone, a signal is sent out by a central facility that addresses a particular receiver unit (beeper) and produces an audible signal. In addition, it may display a number to which the called party should phone back.(Some systems allow a vocal message to be conveyed about the call-back number.) It is then up to the recipient to use the conventional telephone system to call back confirming

\footnotetext{
Present addresses:

1. Xerox PARC, 3333 Coyote Hill Rd., Palo Alto, CA 94304.want.parc@xerox.com

2. Olivetti Research Ltd, 24a Trumpington St.,Cambridge CB2 1QA, England.ah@cam-orl.co.uk

3. Metaphor Computer Systems, 1965 Charleston Rd., Mtn. View, CA 94043.falcao@metaphor.com

4. Sun Microsystems Laboratories Inc., 2550 Garcia Ave., Mtn. View CA 94043.jjg@eng.sun.com
} 
the signal and to determine the required action. Although useful, in practice there are still circumstances where it is not ideal. For instance, if the called party does not reply, the controller has no idea whether they are in an area where the signal does not penetrate, have been completely out of the area for some time, have been too busy to reply, or have misheard or misread the call-back number. Moreover, in the case where there are a number of people who could respond to a crisis situation, it is not known which one is the nearest to the crisis and therefore the most suitable to contact.

An alternative approach is to 'tag' a person and try to locate the tag. The main application of personal tags has been in the area of access control and logging[2]. In many high-security installations, card-key systems restrict access to various parts of the installation. If there are enough access-control zones, the same mechanism can also provide location information on a per-zone basis. For this kind of system, the information is positive and directly available to be acted upon. However, it is inappropriate for most organizations to use access-control techniques to derive location information because of the inconvenience experienced by personnel. There are additional problems arising from groups of people obtaining access to adjoining zones with only one card-key, which is a hard problem to solve.

\section{An Active Badge Design}

A solution to the problem of automatically determining the location of an individual has been to design a tag in the form of an 'Active Badge' that emits a unique code for approximately a tenth of a second every 15 seconds (a beacon). These periodic signals are picked up by a network of sensors placed around the host building. A master station, also connected to the network, polls the sensors for badge 'sightings', processes the data, and then makes it available to clients that may display it in a useful visual form. The badge was designed in a package roughly $55 \times 55 \times 7 \mathrm{~mm}$ and weighs a comfortable $40 \mathrm{~g}$.

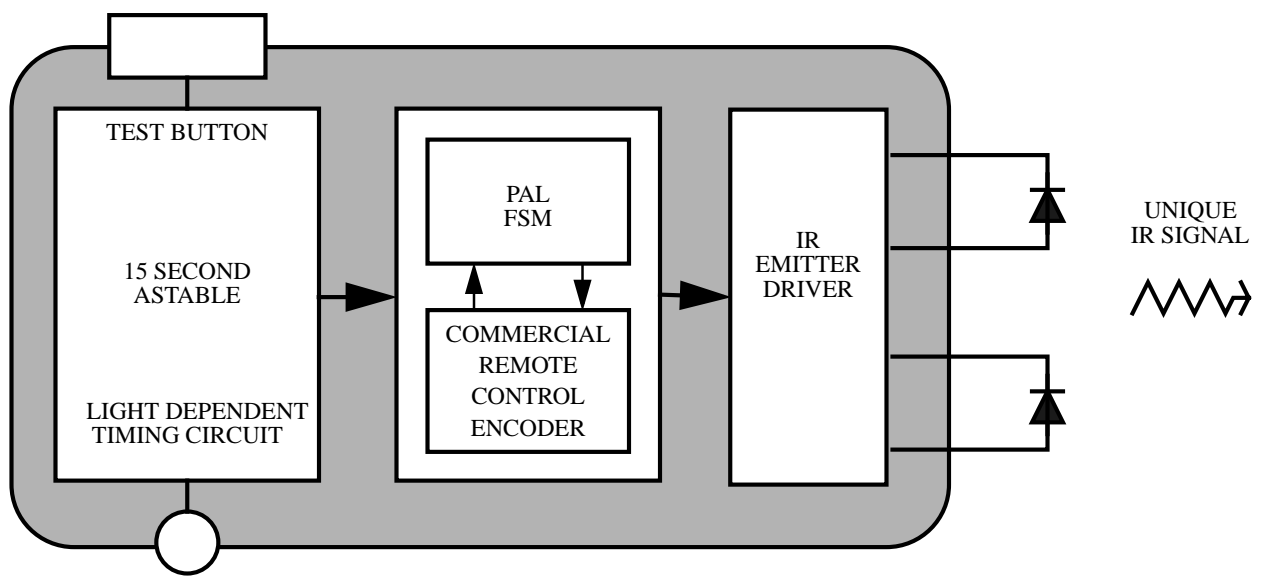

Figure 1: The ORL Active Badge

Pulse-width modulated infrared (IR) signals are used for signaling between the badge and sensor [6] mainly because: IR solid-state emitters and detectors can be made very small and very cheaply (unlike ultrasonic transducers); they can be made to operate with a $6 \mathrm{~m}$ range, and the signals are reflected by partitions and therefore are not directional when used inside a small room. Moreover, the signals will not travel through walls, unlike radio signals that can penetrate the partitions found 
in office buildings. Infrared communication has been used in a number of commercial applications ranging from the remote control of domestic appliances to data backup links for programmable calculators and personal organizers [1]. More recently, IR has been used as the basis for wireless local area networks [3]. Because IR technology has already been exploited commercially, it is inexpensive and readily available for developing new applications such as the Active Badge.

An active signaling unit consumes power; therefore, the signaling rate is an important design issue. Firstly, by only emitting a signal every 15 seconds, the mean current consumption can be very small with the result that 'badge-sized' batteries will last for about one year. Secondly, it is a requirement that several people in the same locality be detectable by the system. Because the signals have a duration of only one-tenth of a second, there is approximately a 2/150 chance that two signals will collide when two badges are placed in the same location. For a small number of people, there is a good probability they will all be detected. Even so, in order to improve this chance, the beacon oscillator has been deliberately designed around low-tolerance components. The components used for the beacon oscillator have a $10 \%$ tolerance rating; for two badges to remain in synchronization for even a single 15-second beacon period, the components would have to be matched better than $1.4 \%$. It is very likely that two badges, which at some instant may be synchronized, will have slightly differing frequencies and thus lose synchronization within a few beacon periods. In practice, synchronization has not been a problem.

The Active Badge also incorporates a light-dependent component that, when dark, turns the badge off to conserve battery life. Reduced lighting also increases the period of the beacon signal to a time greater than 15 seconds. In ambient lighting conditions in a room, this effect only slightly modifies the period, but it is another factor that ensures synchronized badges will not stay synchronized very long. If the badge is placed in a drawer out of office hours, at weekends and during vacation, the effective lifetime of the batteries is increased by a factor of 4 . Note that the more obvious solution of a manual switch was considered a bad idea as it was likely that a badge user would forget to turn it on. Other options for switching the device on included a tilt switch and an accelerometer, although the size limitation of a badge precluded using them in the initial experimental system.

A disadvantage of an infrequent signal from the badge is that the location of a badge is only known, at best, to a 15-second time window. However, because in general a person tends to move relatively slowly in an office building, the information the Active Badge system provides is very accurate.

An Active Badge signal is transmitted to a sensor through an optical path. This path may be found indirectly through a surface reflection, for example, from a wall. A badge must be worn on the outside of clothing, so an essential part of the badge case design was the clip allowing it to be easily attached to a shirt or a blouse. Most commonly, the badge was worn at the breast pocket position; however, some people preferred a belt or waist position. The belt position was not as good when the wearer was seated at a desk but typically the system still detected enough signals to locate the badge.

\section{Badge Sensor and Telemetry-Network Design}

To detect Active Badges in transit through a building, a sensor network must provide thorough coverage through adequate placement and density of sensors. (Because the unit cost of the sensors is low, thorough coverage is not prohibitively expensive.) Sensors need to be placed high up on walls or ceiling tiles of offices and on the entrances and exits of corridors and other public areas. An ideal system would take advantage of existing computer networks as a means of gathering badge sight- 
ings and relaying the data back to a central server for processing. The problem is that many buildings do not have a computer network, and secondly, if a sensor were to be interfaced directly to a modern-day network, the cost of the device would be significantly increased.

A design was conceived that would allow an independent network to support up to 128 sensors, controlled from the RS232 port of any standard workstation. This approach allowed a network of workstations, joined by an Ethernet, to support multiple badge networks, the data being relayed back to one master server by conventional network protocols. The workstations provided a simple connection between each of the badge networks and the main computer network. However, a badge network can also exist on its own supported by a single network controller.
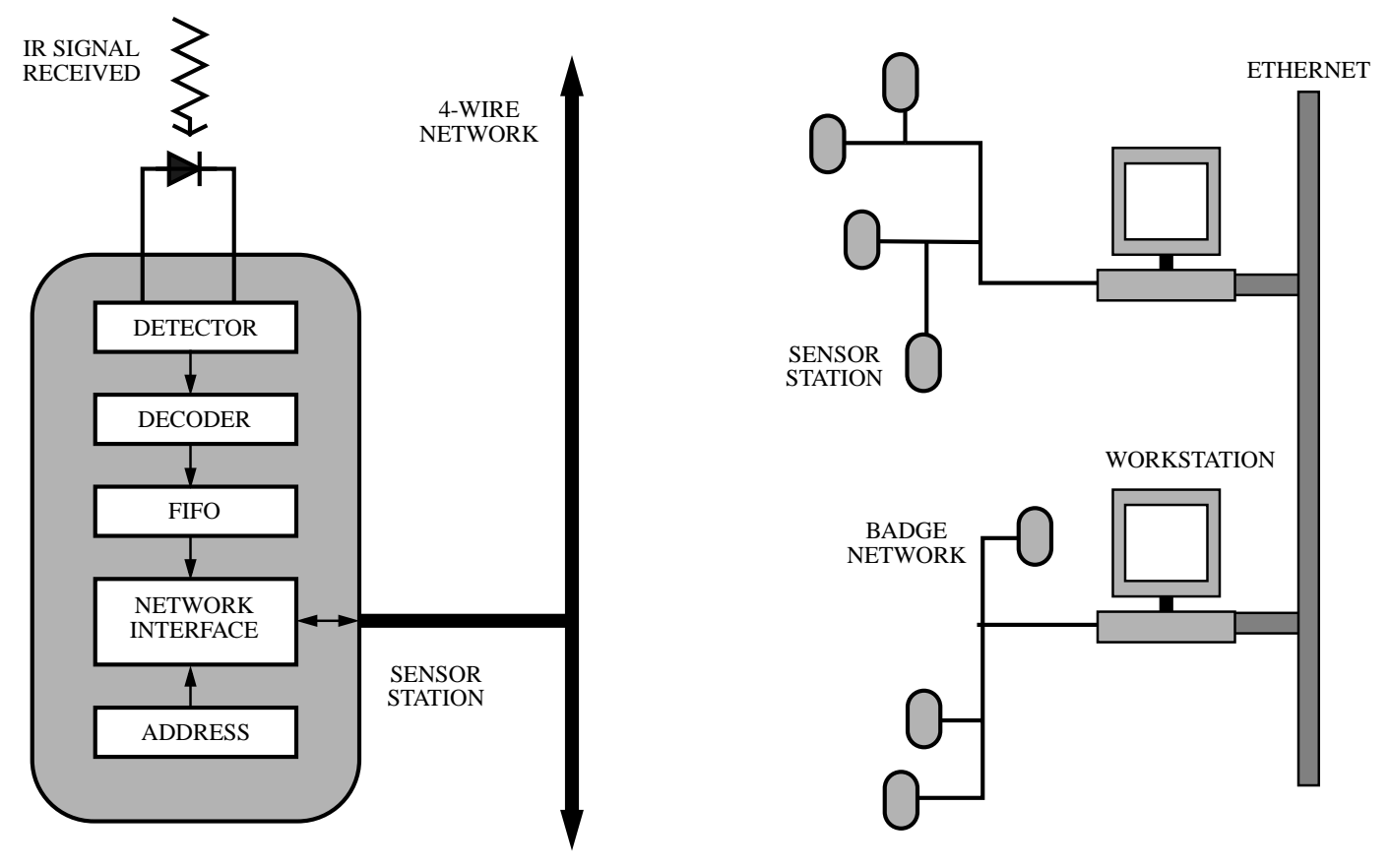

Figure 2: Badge Sensor and Telemetry Network

A prerequisite for the badge network was that it should be able to link all areas of any building with an arbitrary topology. Power would need to be fed though the network because the sensors would be too numerous and distributed in too many remote places for them to be supplied by power locally.

Given these constraints, the badge-sensor network has been designed as a 4-wire system (figure 2). Two of these wires carry the network power-supply, the third carries the serial addressing information allowing the network controller to nominate a station, and the remaining wire carries data back to the network controller. Conventional telephone twisted pairs are used, which means it is possible to take advantage of any spare telephone cable already in a building. Although, as with most networked systems, the cabling is unavoidably a large fraction of the system cost, the cost has been minimized by using standard telephone twisted-pairs. The data-transfer format is logically the same as RS232, but the network is physically a wired-OR system. The consequence is that by using a simple level-shifting interface-box, any computer with an RS232 port can be used as the network master. 
In order that the network master does not have to poll the sensors at high speed to avoid data loss (e.g. if two badges in one room signaled with a very short delay between them), a FIFO has been designed into each sensor that is capable of buffering 20 badge sightings. This allows the network master to multiplex its time between polling the network, manipulating badge data, and making the data available to clients.

\section{An Active Badge Application}

The initial application of this system (the demonstration system) was intended to be an aid for a telephone receptionist. Sensors were mounted in the offices, common areas and major corridors; of course, there are also some areas where people expect to be free from being monitored. The system provides a table of names against a dynamically updating field containing the nearest telephone extension and a description of that location. The format fits onto a standard PC display and is shown in more detail in figure 3. A third field shows the likelihood of finding somebody at that location in the form of a percentage. If it is below 100\%, that indicates the person is moving around, and if they have not been sighted for 5 minutes, the field contains the last time and location at which they were sighted. The last sighted location is still the best clue a receptionist may have to locate somebody and indeed there may be other work colleagues in that area who will know why that person is no longer there. Beyond 24 hours, the last day a badge is sighted is shown in abbreviation; if there are no sightings detected for a week or more, the person is indicated to be 'AWAY'. This format was found to be useful and did not overload the display with too much information. In addition to the display, a command interpreter allows simple investigations to be performed on the system. The commands found to be of most use are listed below:

- $\quad$ FIND (name)

Provides the current location of the named badge and, if it has recently moved, a list of all the locations it has been sighted at in the last five minutes along with the likelihood of finding it at each.

- WITH (name)

Locates a named badge and provides information about other badges that are in the immediate locality of that badge.

- LOOK (location)

Allows an investigation to be made of the badges that are currently near the specified location.

- NOTIFY (name)

An alarm mechanism that generates an audible indication of when the named badge is next sighted after executing the command. 'NOTIFY' is particularly useful when trying to deliver an urgent message to a member of staff who is out of the office on business for long periods of time.

Another useful feature for locating a colleague is a location history mechanism. This command has also been built into the ORL demonstration system.

\section{- HISTORY (name)}

Generates a condensed report of the location history for the named badge during a one-hour period. The system intentionally does not record any location data on a permanent storage medium, to dispel concern about long-term monitoring of an employee's movements. 
The most common use of the system was by the receptionist who routinely used it when forwarding telephone calls from the main switchboard. The receptionist would look at the display of locations and then redirect the telephone call to the correct location in the standard way. After a month of use, the receptionist was so reliant on the system that it was hard to do without it on the rare occasions when the system was not available.

A low-level interface was built to allow certain types of PBX to route telephone calls automatically. The combination of these systems showed proof of concept for a more automatic system, but due to the proprietary nature of PBXs, it was not possible to design a comprehensive solution without the help and consent of a company in the telecommunications business, and that proved difficult to obtain. There are strict regulations for connecting computer equipment to the telephone network in the UK and there was a regrettable lack of interest from UK companies to put in the software effort required to modify the on-site PBX at ORL for the purpose of this experiment. However, PBX's such as the Redcom PBX [4], which is approved for use in the US, have a digital interface designed for computer-integrated telephony, so this would be the most suitable candidate for integration with the Active Badge system if the research continues in the US.

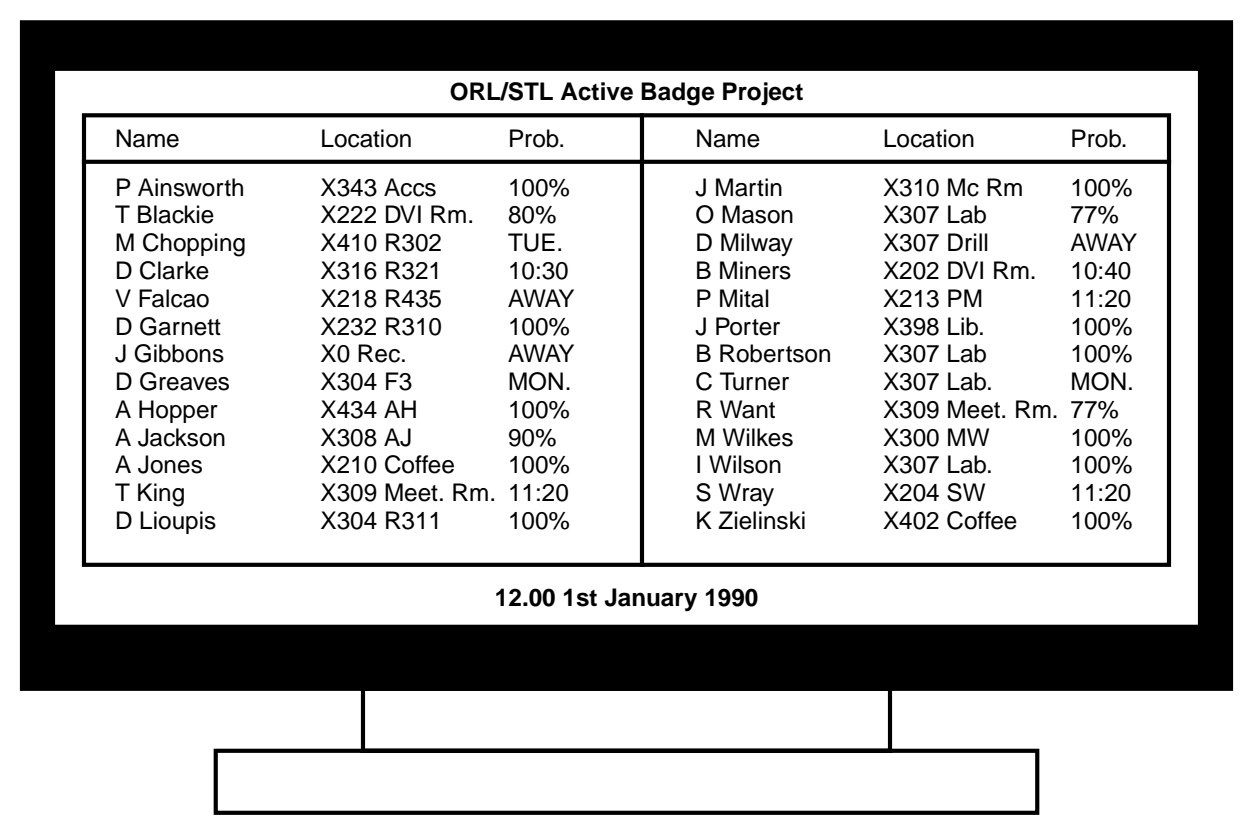

Figure 3: A typical display showing the location of people at ORL

Staff wearing the badges found it useful to have telephone calls accurately directed to their current location but wanted to be able to express more control over when calls were forwarded to them. Where you are, who you are with and what time it is could all be taken into account. For example, most people would prefer not to take unexpected telephone calls when they have just been called into their boss's office; others might not want to receive calls if they are in the lunch room between 12 and 1pm; similarly, a group of people holding an unscheduled meeting in a conference room may not wish to be disturbed. A future application might allow these kinds of conditions to be specified by the user. 


\section{Location Server Design}

The work at ORL has shown that a location server can usefully be thought of as a four-layer system.

- Network Control - The network controller is responsible for polling all sensors on the network. It can be designed to adopt a polling strategy that is biased towards testing sensors that are known to have recently seen badges more frequently than those that have not. Errors in badge-ID format should also be picked up in this layer and erroneous data removed.

- Representation - Once valid data has been extracted from the network, it must be timestamped and entered into a data structure that relates the ID of the badge that has been sighted, location, and time (a triple). In order to obtain information about the recent activities of a badge, a convenient representation can be obtained by building a linked list of these data triples in time order up to a maximum time ( 5 minutes was used for experimentation). For some applications, it is also useful to maintain a list of badges seen at each location.

- Data Processing - An Active Badge network can soon collect a large amount of data. It is necessary to process this data to reflect only the changes in badge locations or provide compressed summaries of the recent history of a badge. If a location server were going to be built as part of a distributed system with the applications running on remote machines, an RPC network interface can be most effectively designed on top of this layer rather than at the Network Control Layer. Some care needs to be put into the server design to compress the location information into a form that does not cause excessive network traffic.

- Display Interface - The Active Badge demonstration-system display uses the location information extracted from the previous three layers as the input for a display function showing textual information about the changing positions of badges. Alternative implementations may choose a graphical display to show the position of badges on a building floor plan using moving icons.

Further enhancements to the system could be considered if the server had a component that knew about the layout of a building and the sensors within it. Such a system might be able to make simple predictions. For instance, given the time and the previous locations at which a badge has been detected, it may be possible for the system to predict the final destination of a person who is moving about the monitored area and so provide more useful display information. Another enhancement might also be to take advantage of information found in on-line diaries to be able to show on the display the purpose and duration of meetings in progress.

\section{The Initial System Trial}

A location system has many advantages, but it also raises many social fears. When most people learn that a personnel location system may be installed in their workplace, their immediate reaction is one of horror. Putting such a device into the work-place is viewed unfavorably on the grounds that it violates personal freedom and individual privacy rights. Before the initial trial period at ORL, the reaction was one of concern. ORL is a research laboratory containing 32 staff, three of whom are administrators and six are contractors. There is one Laboratory Director and a Laboratory Manager; the remainder are engineers. Management requested that everybody wear the badges for a trial period of two weeks, and from then on it was considered optional. In the initial 10 workdays, the incidence of telephone calls not reaching the correct person dropped substantially; the main laboratory no longer received a continuous stream of disruptive enquiry calls, whereas the 
main laboratory had previously been the default place to check for anybody if they were not in their own office. ORL's receptionist had a much easier time and was able to avoid many wasted trips, up and down corridors, trying to find members of staff. Also the perception of clients telephoning ORL was one of good organization since the receptionist was able to say with great certainty where somebody was or when they were last seen or the likelihood that they had just taken a lunch break, all without the need to be explicitly informed by the staff. It also gave employees the advantage that when expecting a telephone call they could wander around the laboratory, the printer room, or the coffee room with confidence that the call would still reach them successfully. Groups of people who regularly wanted to hold meetings could find each other easily with very little notice since they could also access the location information. Other advantages from a security and convenience point of view were that visitors could easily be located during their visits to ORL. Finally, in the friendly environment of a research laboratory, any initial fears the staff had about management abuse of the system proved to be unfounded. For all of these reasons, the two-week trial was a success, and people continued to wear their badge quite willingly after this period.

\section{The Current Cambridge UK Installation}

The prototype system was first installed in February, 1990, and now consists of over 100 badges, 200 sensors, and 5 badge networks in use at four sites in Cambridge, England. Although some badges have had to have their batteries replaced, others are still operating after 16 months in service. The system is in use at two organizations: ORL and the University Computer Laboratory. All the sites are within a few minutes' walk of each other, and faculty members and ORL staff frequently move between buildings. The Active Badge location system spans all four sites, and information about any person's whereabouts is potentially available from any terminal on the network. This includes local telephone extension numbers or external numbers where needed. Access to the data is restricted: for example, undergraduate students cannot see the location of arbitrary Olivetti staff members, and the way the data is protected has proved acceptable to most users. It is sufficiently popular that there are often requests to extend the system to cover more locations within the University.

\section{Privacy Issues}

Despite the success of the Cambridge installation, the majority of people who first learn about the system are not convinced it is something they would like in their own work situation. The privacy issues are clearly very important. An observation made at ORL is that the system has to be worked with for a period of time before it can be judged with a clear understanding of the issues. The following observations should be made.

Firstly, there will always be some days when for whatever reason somebody does not wish to be located. The location system tracks badges and NOT people. Anybody wearing a badge can remove it and leave it on a desk. The Active Badge system will then be misled into locating that person in an area that has been chosen for this deception. This kind of escape mechanism is not an undesirable system feature and may be an important factor in making this system acceptable for common use. In practice, at ORL this was not known to be a common use of the system.

Secondly, it is the way location data is used or logged that is often the main concern for badge wearers. It is possible for a locator system to be devised that allows each person to specify who may locate them and in addition provide information about who has tried to locate them and how often. 
If this information is valid, it would soon become clear when the system was being misused. Such privilege systems are well understood and are in common usage to protect computer filing systems. It may be that despite knowing the system can be made secure, there will always be a fear that a secret logging system has been installed by management. A company that has a bad management policy can, of course, make life unpleasant for employees with or without a badge system, and this is a different kind of problem. Simple examples of other potential location devices already in place in the office that are open to abuse are the network computer which can keep track of login times and keystrokes per minute, PBX's which can be used to monitor call set up and provide location information about time spent at your desk, and card-key systems which give away location information about movements within controlled areas. In practice, it is rare that these techniques are used against employees, but it is true that each of these systems potentially makes it easier for a misguided management to abuse the day-to-day privacy we all expect.

The problem is that technology in itself is rarely inherently bad; it is just that it can be used for good or bad purposes. We are living at a time when it feasible to build an effective location system at low cost, and we should work to ensure that this kind of technology is used to the advantage of employees, rather than to compromise their privacy. If it turns out that location systems in our society at large are abused, then the rights of individuals must be protected. In this case legislation must be drawn up to ensure a location system cannot be misused, while still allowing us to enjoy the benefits it brings. Such legislation may prevent an employer from insisting that employees wear locators and prevent location-data logs from being used as the basis for productivity assessment or as the only grounds for a dismissal.

\section{Conclusions and Future Directions}

The Active Badge system at ORL has been widely accepted by the members of staff and is used on a daily basis for either locating people for meetings or for transferring telephone calls to the correct person. Observation of the system shows that a PBX controlled directly by the badge system with the option for human intervention would be of considerable use to office support staff. There have now been four additional installations of the badge system: at Olivetti STL, Xerox EuroPARC, MIT Media Laboratory and Xerox PARC. Invariably, they have all had initial concerns about privacy; however, while it has generally been the case that employees are very concerned about the system at the time of installation, the concern considerably diminishes after they have used the badges for a short period of time. In practice, most users find the system has many benefits and that the information provided by the system is not abused in these environments.

The Active Badge system is a technology-driven research project. The enabling technology has been small low-cost IR emitters and control logic designed for the remote control requirements of consumer products such as televisions. The most important result of this work is not, "Can we build a location system?", but "Do we want to be part of a location system?" There is a danger that in the future this technology will be abused by unscrupulous employers. If it is, then legislation must be used to protect us from this kind of technology abuse. However, it is our conclusion that amongst professional people responsible for their own work time, it is a very useful and welcome office system.

The current badge is a device that is limited to providing location information, but with the use of more complex miniature devices such as ASIC technology or low-power microcontrollers, a great deal more can be achieved. Two-way communication is one way to extend functionality. A badge 
that has a display and can receive data can also operate as a pager. An Active Badge in combination with a pager provides the best of both location techniques.

A transponding badge can also be used as a key, authenticating itself upon entering a secure area. The problem with a remotely operated key is that the signals it sends can be recorded and played back later to masquerade as the same key. By using a randomized challenge/response protocol from the secure area to the key, good protection can be given against this kind of forgery. The unforgeable key can of course be stolen, but so can a conventional key; yet a badge offers additional advantages. The basic locator-badge without additional features can also be used in combination with conventional security systems such as motion detectors. Motion detectors warn about all movement that occurs in a building, but the badge system allows a refinement indicating where there is movement accompanied by a badge ID. Movement without detecting an Active Badge could alert security personnel to a suspicious situation.

A future badge may also be able to signal several different codes, for instance: a location code, a battery-low code, and codes generated by pressing buttons on its case. The button codes may signal special commands to the controller that modify its behavior. In the case of telephone call-forwarding a button-press received from a meeting room may stop calls from being forwarded there. However, if the badge is seen in another room (i.e. the meeting has finished), the command is now cancelled automatically. This example illustrates an important aspect of badge technology: a signal produced by an Active Badge can have different effects at different locations on the network.

Where the Active Badge concept is used and becomes accepted as an office system, it may be combined with other building management control functions such as: fire alarms, security, heating, airconditioning and lighting control. The Active Badge extends the concept of an integrated building to take into account the location of personnel in that environment.

\section{Acknowledgments}

The authors wish to thank Carl Dellar for supporting the project in its early phase, Roger Needham and David Wheeler for their valuable advice, and Martin Simmons and Pradeep Mital for their help prototyping the original Active Badge demonstration system.

\section{References}

[1] Harper, S. L., Worsley R. L., and Stephens, B. A.,An Infrared Link for Low-Cost Calculators and Printers. Hewlett-Packard Journal, October 1987.

[2] Hewkin, P., Smart Tags - The Distributed Memory Revolution. IEE Review, June 1989.

[3] Paepcke, A., Crawford, R.D., Jamp, R., Freeman, C. A., and Lee, F.J., Chipnet: An Optical Network of Terminals and Workstations. Elsevier Science Publishers B. V. (North-Holland), Computer and ISDN Systems 15 (1988) pp. 203-215.

[4] Redman, B. E.,A User Programmable Telephone Switch, EUUG Conference, Helsinki, May 1987.

[5] Ronayne, J., The Integrated Services Digital Network: from Concept to Application. Pitman Publishing, London UK, 1987.

[6] Satellite, Cable and TV IC Handbook. Plessey Semiconductors, 1988 pp. 64, 67, 124 